\title{
Use of Beam Probes for Rigidity Calibration of the A1900 Fragment Separator
}

\section{AUTHORS}

T. N. Ginter ${ }^{1}$, F. Farinon ${ }^{2} *$, T. Baumann ${ }^{1}$, M. Hausmann ${ }^{2}$, E. Kwan ${ }^{1}$, O. Naviliat Cuncic ${ }^{1}$, M. Portillo $^{2}$, A. M. Rogers ${ }^{1} \dagger$, J. Stetson ${ }^{1}$, C. Sumithrarachchi ${ }^{1}$, A. C. C. Villari ${ }^{2}$, S. J. Williams ${ }^{1}$

${ }^{1}$ National Superconducting Cyclotron Laboratory, Michigan State University, East Lansing, MI 48824, USA

${ }^{2}$ Facility for Rare Isotope Beams, Michigan State University, East Lansing, MI 48824, USA

* Present address: GSI, Planckstrasse 1, D-64291 Darmstadt, Germany

$\dagger$ Present address: Department of Physics, University of Massachusetts Lowell, Lowell, MA

01854, USA.

\section{ABSTRACT}

Use of a beam-based approach is presented for establishing a rigidity calibration for the A1900 Fragment Separator located at the National Superconducting Cyclotron Laboratory. Also presented is why an alternative approach to the rigidity calibration - using detailed field maps of individual magnetic components - is not a feasible basis for deriving an accurate calibration. The level of accuracy achieved for the rigidity calibration is $\pm 0.1 \%$.

\section{KEYWORDS}

Electromagnetic Separator; Rigidity; Calibration; Dipole Magnet; Fragmentation Reactions

\section{INTRODUCTION}

The A1900 fragment separator [1] is a key component of the Couple Cyclotron Facility (CCF) of the National Superconducting Cyclotron Laboratory at Michigan State University. The A1900 provides in-flight separation of rare isotopes produced in fragmentation reactions for delivery to downstream experiments. Figure 1 shows a schematic view of the A1900 highlighting the target position (where accelerated stable heavy ion beams interact with a target to produce rare isotopes), the four $45 \square$ bending dipoles D1-D4, the three dispersive image positions, I1, I2, I3, and the achromatic focal plane position marked FP. The A1900 also consists of 24 quadrupole elements arranged in 8 triplets; 16 of the quadrupoles also incorporate concentric sextupole and octupole elements to provide higher order corrections. All of the magnetic elements are superconducting. 


\section{Use of Beam Probes for Rigidity Calibration of the A1900 Fragment Separator}

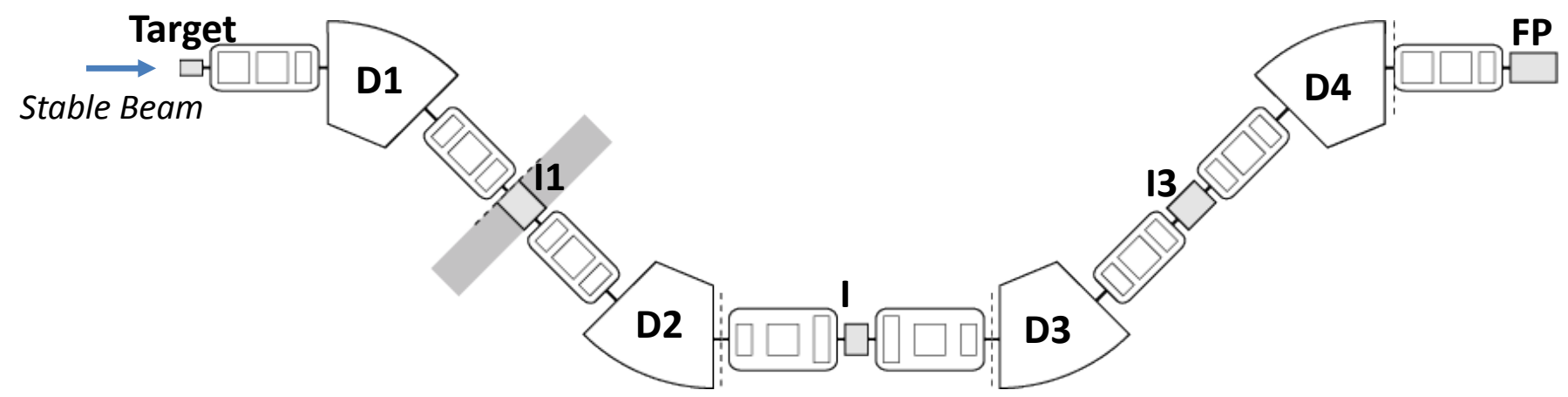

Figure 1. Schematic layout of the A1900 fragment separator.

The rigidity setting of a magnetic fragment separator is used in selecting specific rare isotopes, and it is an important input for some experiments and for efficient beam stopping. The setting is defined in terms of the rigidity of beam particles that are deflected along the central axis. A good rigidity calibration for a separator means that the rigidity of isotopes leaving the device is accurately defined by the rigidity setting.

Two general approaches are used for calibrating the rigidity of a separator: (1) empirical - based on the response of calibrated beam probes passing through the system, and (2) theoretical based on modeling the response of beam to the system from beam dynamics.

For the A1900 the main challenge with an empirical approach is that calibrated beam probes are not readily available. The best available probes are the primary beams from the CCF. However, not only is it not clear what the accuracy is for the nominal rigidity of these beams, but also the available beam rigidities cover only part of the A1900 operating envelope. The primary beams range in rigidity from 3.5 to $4.5 \mathrm{Tm}$, while the operational range of the A1900 extends from 1 to $6 \mathrm{Tm}$.

The challenges associated with using a theoretical approach to extract an accurate calibration are even more problematic. Use of a simple model, assuming ideal dipole behavior and that the central beam follows the optic axis through the separator, is out of the question. One issue is alignment - both in terms of inaccuracies in the positioning of the magnetic components within the A1900 and in terms of uncertainties in the positions of reference points we use for setting up the beam. A second issue is the deviation of dipole performance from ideal behavior. The assumption that a dipole acts strictly in first-order - that the deflection of particles by rigidity varies linearly with dipole field - is not precisely correct. A third issue comes with the use of sextupole correction elements. Each sextupole has a dipole component to its field [2], which ensures that the "central" beam can never actually follow the optic axis.

Use of a more sophisticated theoretical approach for determining an accurate rigidity calibration - based on modelling from measured field maps of the magnetic elements - is also problematic. As discussed below under results, the field maps available for the A1900 components are not detailed enough to be used readily for this purpose. Alignment uncertainties are again another concern. Yet another concern is the field environment; for the $\mathrm{CCF}$, fringe fields from the 


\section{Use of Beam Probes for Rigidity Calibration of the A1900 Fragment Separator}

cyclotrons are not negligible in the first part of the A1900 and can change the dipole field readings at the level of $0.02 \%$ for $\mathrm{D} 1$ and $0.01 \%$ for $\mathrm{D} 2$.

Based on these considerations we have chosen an empirical approach for the A1900 rigidity calibration because it accounts for perturbations that we cannot adequately model or control. To obtain beam probes at rigidities lower than those available from the CCF's standard beams, we employed degrader foils of various thicknesses at the A1900's target position to reduce the beam energy. To measure the rigidity of each beam we used as a probe, we employed a time-of-flight technique [3] downstream of the A1900. A full description of our efforts to calibrate the A1900, including the time-of-flight measurement and error analysis, will be presented in a forthcoming publication. The rest of the present discussion is restricted to describing how these beam probes are used to extract a rigidity calibration.

\section{METHODS}

The goal was to map the field vs. the particle rigidity dependence for each dipole in the context of the A1900 as installed and operated. This was achieved for each beam probe by careful beam centering at the main reference locations within the A1900: target, I1, I2, I3, FP (see Fig. 1). These are where we center the beam during normal operation and where we use selection slits to define the rare isotope settings. The resulting data make it possible to determine from future (and past) dipole field readings the rigidities of particles being passed through each part of the A1900.

Table I lists the four CCF beams used for the rigidity calibration. Two types of beam probes were used: undegraded beam (i.e., not passing through degrader foils at the A1900 target position) and degraded beam. The use of degrader foils shifted the charge state of beams that were not already fully stripped to (predominantly) the fully-stripped charge state. In the case of ${ }^{96} \mathrm{Zr}$, degrader foils also populated other charge states at a level strong enough for use in the rigidity measurements.

Table I. Overview of CCF beams used for A1900 rigidity calibration.

\begin{tabular}{|c|c|c|c|c|}
\hline Isotope & $\begin{array}{c}\text { Energy from } \\
\text { Cyclotron } \\
{[\mathrm{MeV} / \mathrm{u}]}\end{array}$ & $\begin{array}{c}\text { Charge state } \\
\text { From Cyclotron }\end{array}$ & $\begin{array}{c}\text { Maximum } \\
\text { Rigidity } \\
{[\mathrm{Tm}]}\end{array}$ & $\begin{array}{c}\text { Charge States } \\
\text { From Target Used }\end{array}$ \\
\hline${ }^{82} \mathrm{Se}$ & 140 & $32+$ & 4.52 & $34+$ \\
\hline${ }^{124} \mathrm{Sn}$ & 120 & $45+$ & 4.48 & $50+$ \\
\hline${ }^{96} \mathrm{Zr}$ & 120 & $37+$ & 4.22 & $40+, 39+, 38+$ \\
\hline${ }^{48} \mathrm{Ca}$ & 140 & $20+$ & 4.24 & $20+$ \\
\hline
\end{tabular}

The procedure for setting up each beam probe through the A1900 consisted of first accurately setting the undegraded CCF beam position and angle at the target location just as these parameters would normally be set for any A1900 setting involving primary or rare-isotope beams. The beam position at the target location can be controlled to within $\pm 1 \mathrm{~mm}$ in the A1900's dispersive direction using a viewer. The beam size at this position is typically below 2 $\mathrm{mm}$ in diameter. A selection of beryllium or aluminum foils in a variety of thicknesses mounted 


\section{Use of Beam Probes for Rigidity Calibration of the A1900 Fragment Separator}

on the main target ladder drive or on two target ladder drives just upstream could be used alone or in combination to degrade the beam to lower energies.

A slit in the dispersive direction at I1 was closed to its minimum setting of $\pm 1 \mathrm{~mm}$ (equivalent to a momentum spread of $\pm 0.03 \%$ ). In the case of the degraded primary beams, the momentum spread of the beam is typically much wider than the Il gap because of energy straggling through the degrader(s). For these beams the rigidity of the separator was set based on the nominal values for cyclotron beam energy and degrader thickness and then adjusting the A1900 rigidity settings (and hence also the D1 field) up or down slightly as needed to pass the beam through the I1 gap. We did not spend time trying to carefully center the beam distribution at I1 on the slit gap - it was enough to have a reasonably intense portion of the distribution passing through the gap. Thus, the exact rigidity for these beams was defined by the setting of D1 rather than by the exact degrader thickness. In the case of the undegraded primary beams, all A1900 magnets were scaled to the nominal rigidity of the primary beam, and only the field of dipole D1 was adjusted to center the beam (with a typical momentum spread of about half the opening of the I1 slit) within the gap.

For the $\mathrm{Zr}$ beam in most of the cases where we used a fully-stripped degraded beam as a probe, we were able to find a thicker combination of the installed degrader materials that populated a non-fully-stripped charge state of the beam that also reached the slit gap at I1. In this way we were able to obtain a new beam probe at the same rigidity but with a lower energy (and, hence, longer time-of-flight) simply by switching among degraders while skipping the lengthy process of setting up a new beam probe through the separator at a different rigidity. We still used the time-of-flight measurement downstream of the A1900 with this lower energy beam probe to obtain an independent measurements of the A1900 rigidity setting.

Once a beam probe was set up at I1, the fields of dipoles D2, D3, and D4 were sequentially adjusted to position the beam carefully at the respective reference points I2, I3, and FP. In most cases slits downstream of the I1 position were closed as tightly as possible in the dispersive direction (1 $\mathrm{mm}$ at $\mathrm{I} 2,2 \mathrm{~mm}$ at $\mathrm{I} 3$ ) to define the rigidity of the beam as much as possible.

Dispersive and non-dispersive slits at the FP were closed to gaps of $1 \mathrm{~mm}$ each to reduce the beam spot size for the downstream time-of-flight measurement. Use of narrow slit settings ensured that only beam particles along a strictly-defined path were used for the downstream time-of-flight-based rigidity measurements since the beam would otherwise be lost if it shifted or some A1900 element drifted during that measurement.

\section{RESULTS AND DISCUSSION}

Figure 2 shows the results of the rigidity calibration for dipole D4. Dipoles D1-D3 show a similar behavior. The two measured quantities that determine its performance are magnetic field (needed to keep the beam positioned correctly) and beam rigidity (from the time-of-flight measurement). We plot D4's behavior, however, in terms of the bending radius $\rho$ (beam rigidity/field) vs. beam rigidity to show better its deviation from ideal dipole behavior (a horizontal line). As can be seen from the spread in the plotted data points, the calibration is precise to a level of $0.1 \%$ in rigidity. 


\section{Use of Beam Probes for Rigidity Calibration of the A1900 Fragment Separator}

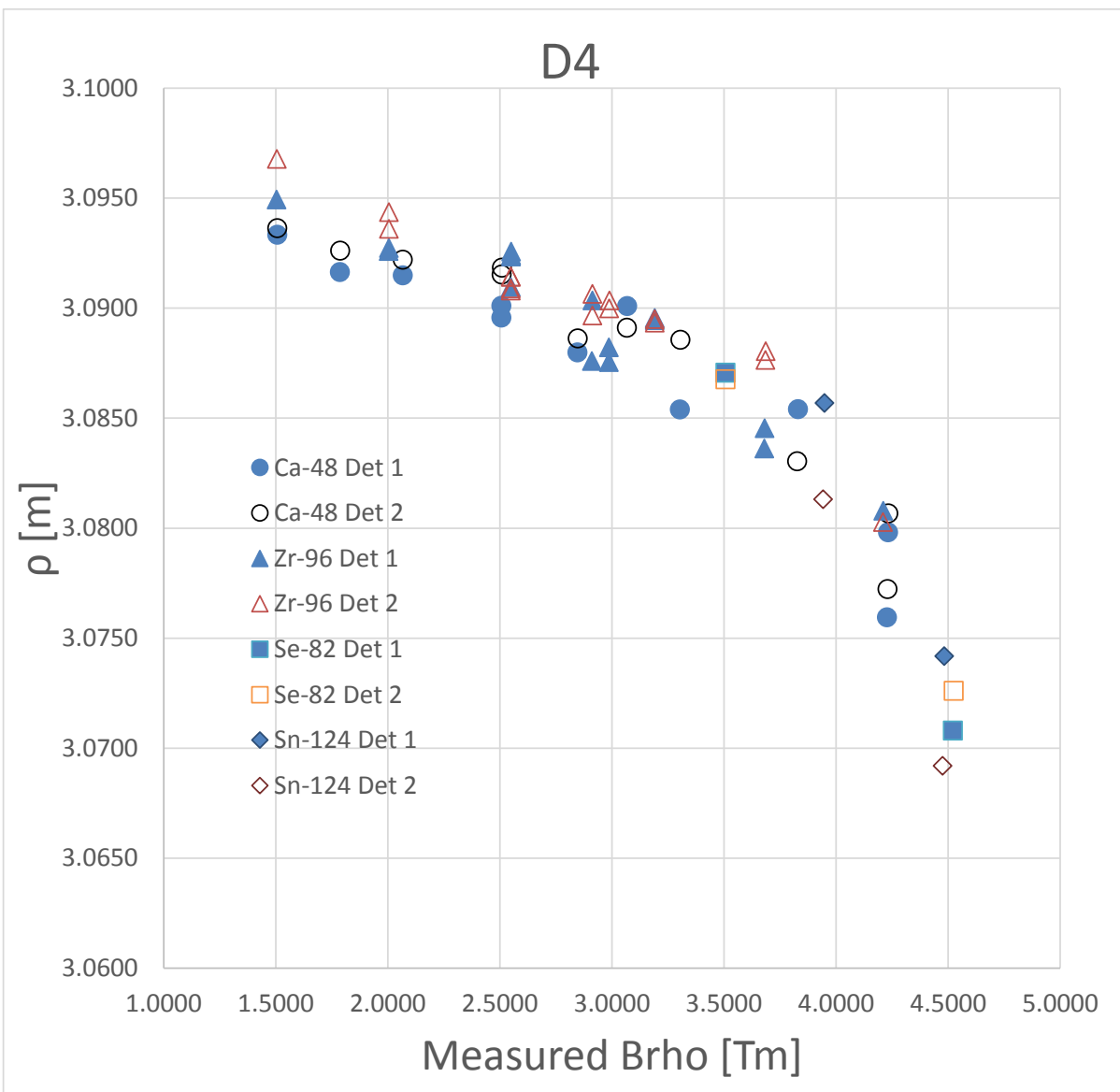

Figure 2. Bending radius $\rho$ plotted as a function of beam rigidity for dipole D4 from data obtained for the rigidity calibration. "Det1" and "Det2" indicate results from two independent detectors used in the time-of-flight-based rigidity measurement.

Keep in mind that the new calibration is valid strictly only within the context of the standard ionoptical solution that we use for most applications and which we used for the calibration. Also note that the calibration only covers rigidities up to the highest $\mathrm{CCF}$ beam rigidity used for the calibration. Another approach is needed to address higher rigidities accurately; such rigidities are used for only a small part of A1900 operation.

The results obtained for this rigidity calibration are consistent with information from field maps made before the dipoles were installed within the A1900. For example, $\rho$ decreases with rigidity, and $\rho$ values obtained for dipole D3 are smaller than those for the other dipoles. Attempts, however, to match the observed dipole behavior in this work to the earlier dipole field maps in detail have been unsuccessful. There are several possible contributing factors to the difficulty in lining up the data sets: insufficient density of points in position and in field settings in the original field map data, uncertainties in the reference for positions in the field map data, uncertainties in the positions of the dipoles within the A1900, and uncertainties in present positioning of NMR probes used for A1900 field measurements. It might be possible to link the dipole field map data to the beam-based rigidity calibration data through a more thorough modelling of the dipoles based on the field map data. 


\section{Use of Beam Probes for Rigidity Calibration of the A1900 Fragment Separator}

The method previously employed for the rigidity calibration was

1) Assume the nominal rigidity of the undegraded CCF beam to be accurate.

2) Determine the corresponding fields of dipoles D1-D4 by positioning the beam at the target and the 4 image positions as described above.

3) Calculate an "effective" bending radius $\rho$ for each of the four dipoles from their field readings and the nominal rigidity.

4) Use these effective $\rho$ 's to scale the dipole fields linearly to any settings from this CCF beam used for rare-isotope production or beam delivery.

New dipole effective $\rho$ 's were determined for every CCF beam change since the values never quite repeated with repeat instances of the same CCF beams - probably because of inaccuracies in beam positioning and slight variations in beam energy.

In consideration of the data in Figure 2 and of the fact that most rare isotope settings are made at a rigidity lower than that of the undegraded CCF beam, this approach typically results in quoting a rigidity that is low compared to the actual rigidity corresponding to a dipole field setting. Figure 3 shows a plot based on D3 and D4 field readings collected from production settings before the A1900 rigidity recalibration where these dipoles were both set to the same rigidity values. The open squares show the difference in D4 rigidity as determined from the old and new calibrations. As expected, the rigidities from the old calibration are generally lower compared to the new calibration - with an average difference of $-0.11 \%$ (just over the error limit of $0.1 \%$ for the new calibration). 


\section{Use of Beam Probes for Rigidity Calibration of the A1900 Fragment Separator}

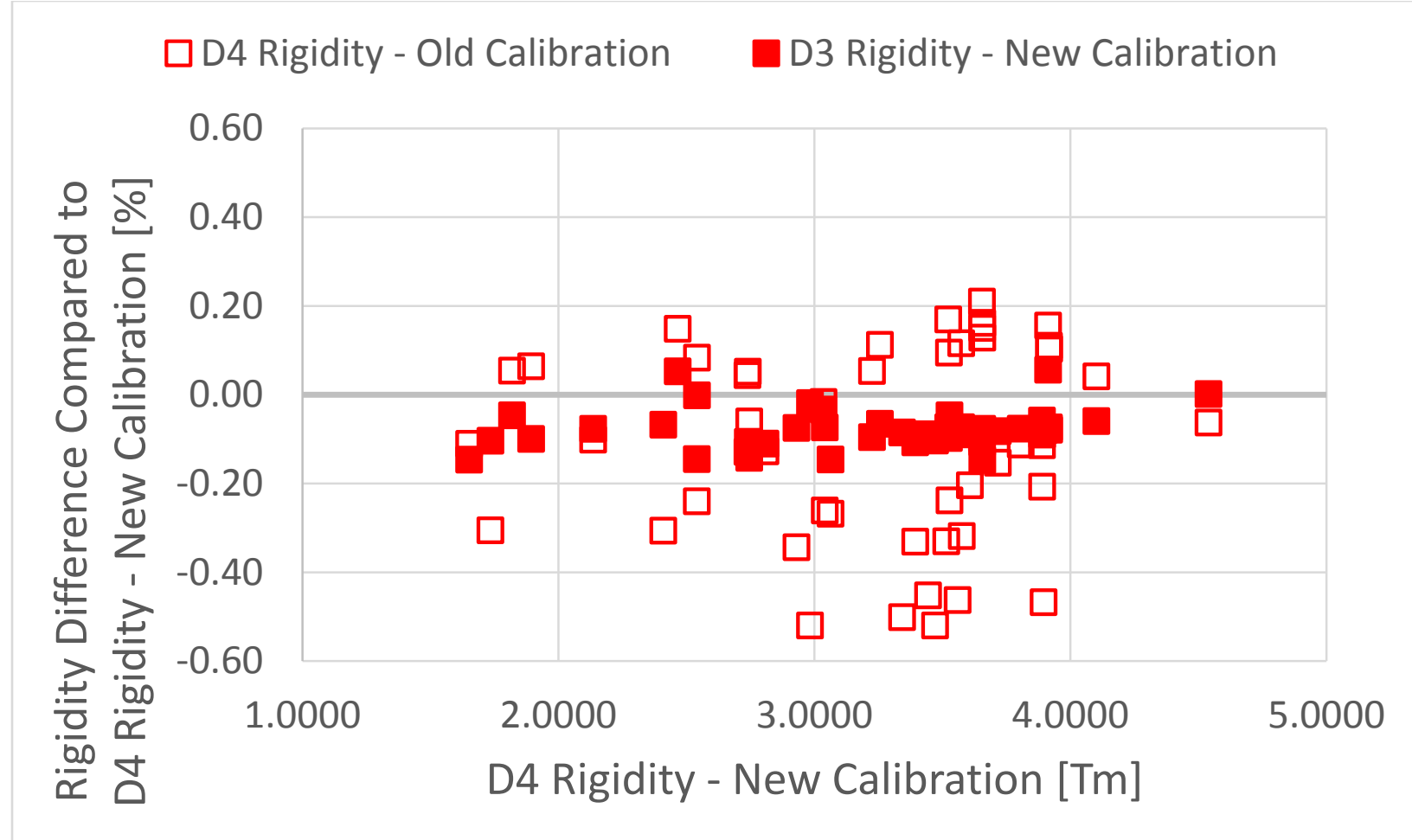

Figure 3. Sample data calculated from D3 and D4 field readings in production settings from before the A1900 rigidity recalibration. The plot shows the consistency between the rigidity from D3 and D4 based on the new calibration (filled squares), and it shows the spread in the old rigidity calibration compared to the new one (open squares compared to filled squares).

The closed squares in Figure 3 show the difference in rigidity from the new calibration of D3 compared to D4. There is less spread in this data set compared to the data set with open squares. This result points not only to the improvement in the new calibration compared to the old one but also to the consistency between the rigidities determined for $\mathrm{D} 3$ and $\mathrm{D} 4$ under the new regime the standard deviation is $\pm 0.04 \%$, much lower than the spread of $\pm 0.22 \%$ for the open squares.

\section{CONCLUSION}

We have presented the difficulties of using an approach based on modelling to establish an accurate rigidity calibration for the A1900 fragment separator. We have described an empirical approach to the A1900 rigidity calibration that makes use of the as-built layout of the A1900 and is tailored to our operational protocols. We have achieved a calibration with an accuracy of $\pm 0.1 \%$ in rigidity over a range from $1.5-4.5 \mathrm{Tm}$ covering most of the typical range of operation. 


\section{Use of Beam Probes for Rigidity Calibration of the A1900 Fragment Separator \\ ACKNOWLEDGEMENTS}

This work is supported by the U. S. National Science Foundation under grant No. PHY-11-

02511 and the U.S. Department of Energy under cooperative agreement DE-SC0000661.

\section{REFERENCES}

[1] D. Morrissey, B. Sherrill, M. Steiner, A. Stolz, and I. Wiedenhoever, Nucl. Instr. and Meth. B 204, 90 (2003).

[2] M. Portillo, et. al, Nucl. Instr. and Meth. B 317, 271 (2013).

[3] J. M. Casandjian, et al., Nucl. Instr. and Meth. A 334, 301 (1993). 